\title{
Periodic pattern formation in the coupled chemotaxis-(Navier-)Stokes system with mixed nonhomogeneous boundary conditions - CORRIGENDUM
}

\author{
Chunhua Jin \\ School of Mathematical Sciences, South China Normal University, \\ Guangzhou 510631, China
}

Keywords: chemotaxis-fluid system; mixed boundary; time periodic pattern; classical solution

In the above paper (Jin, C. (2020) the author's affiliation was incomplete. The author apologises for this error. The affiliation should read;

School of Mathematical Sciences, South China Normal University, Guangzhou, 510631, China

This has been updated in the original article

\section{Reference}

1 Jin, C. (2020). Periodic pattern formation in the coupled chemotaxis-(Navier-)Stokes system with mixed nonhomogeneous boundary conditions. Proceedings of the Royal Society of Edinburgh: Section A Mathematics, 150(6), 3121-3152. doi:10.1017/prm.2019.62

(C) The Author(s) 2021. Published by Cambridge University Press on behalf of The Royal Society of Edinburgh 